\title{
Farmers' Awareness towards Drip Irrigation System and Flood Irrigation System in Coimbatore District
}

\author{
Dr. (Mrs.) P. Maheswari \\ Assistant Professor in Commerce (CA), CBM College, Kovaipudur, Coimbatore-42, Tamilnadu, India
}

\begin{abstract}
Agriculture is the backbone of Indian Economy and a key sector in India. Agriculture is the largest user of water which consumes more than 80 per cent of the country's exploitable water resources. The conventional methods of water conveyance and irrigation are highly inefficient due to wastage of water and other clogging problems. In this context, the use of modern irrigation methods like drip irrigation and sprinkler irrigation are the only alternative for efficient use of surface as well as ground water resources. Hence, in this chapter, the meaning, Significance, components, scope of Drip Irrigation are discussed, apart from describing the origin, need, types of irrigation.
\end{abstract}

Keywords: Agriculture

\section{Drip Irrigation}

Drip irrigation method (DIM) is a technical measure introduced about to decades back to increase the water use efficiency in Indian Agriculture. The increasing demand for irrigation water coupled with a rapid decline in net potential of water available in the recent years has forced adoption of new methods of irrigation for crop cultivation all over the world. In this method water will be supplied constantly or at regular interval at the root zone of crops through a network of pipes with the help of emitters. It is well known fact that the DMI is mainly used for water saving. The consumption of water is less when it is applied by pipe network as it is completely stops the evaporation and distribution losses of water ${ }^{1}$.

\section{Drip Irrigation in India}

In India, it was introduced in the early seventies, and during the last few years this system has started gaining momentum. The adoption of the drip system started in areas having water scarcity, poor quality water, and undulating terrains. The Indian National Commission on Irrigation and Drainage, in its report (INCID, 1994.) has reported that in India, 70,859 ha area are under drip irrigation, which is 3.97 per cent of the area irrigated by drip irrigation systems worldwide.

The scientists at the Tamil Nadu Agricultural University (TNAU), Coimbatore, who are considered to be the pioneers in drip irrigation research in India, have conducted largescale demonstration in the farmers' field for various crops, which received encouraging response from the farmers (INCID, 1994). However, the adoption of drip method of irrigation was very slow till mid-eighties mainly because of lack of promotional activities from the State and Central governments. The formation of the National Committee on the Use of Plastics in Agriculture (NCPA) by the Ministry of Petroleum, Chemicals and Fertilisers, Government of India, during 1981 under the Chairmanship of Dr. G. V. K. Rao is termed as the first milestone for the development of microirrigation in India (GOI, 2004). With the establishment of 17 different Plasticulture Development Centers (PDCs) across different agro-climatic regions in the country, the NCPA has played a crucial role in the technological development of micro-irrigation in India. Besides recommending policy measures to the government, the NCPA also played an important role in promoting drip method of irrigation through conducting seminars focusing on micro-irrigation (GOI, 2004).

\section{Profile of the Study Area - Coimbatore District}

Tamil Nadu, in India, has been divided into five agricultural zones based on the agro climatic conditions and soil profile. Coimbatore district falls under Zone II. where Coimbatore is the third largest city in the state, and is popularly known as textile capital of south India. It is situated on the banks of the river Noyyal.

Objectives of the study

1) To evaluate the crop yield between surface irrigation and drip irrigation.

2) To study drip irrigation system controls pests and diseases.

Hypotheses of the study

Ho1:Agricultural Profile and Opinion that Drip Irrigation Increases Yield

Ho2:Agricultural Profile and Opinion that Drip Irrigatio System controls Pests

\section{Methodology}

\section{Sources of Data}

The study is mainly based on primary data, which are collected from farmers through Interview Schedule consisting of 42 questions, of which, 11 questions were related to the personal profile of the respondents, 8 questions were related to Agricultural Profile and the rest were related to Irrigation Profile of the respondents .The secondary data needed for the study were collected from the Department of Agriculture, Government of Tamil Nadu, Suppliers of drippers and online sources, apart from the related journals, articles, and books. 


\section{International Journal of Science and Research (IJSR) \\ ISSN (Online): 2319-7064 \\ Index Copernicus Value (2013): 6.14 | Impact Factor (2015): 6.391}

\section{Sample Design}

The primary data were collected from 395 farmers in Coimbatore, District, in Tamil Nadu. Coimbatore District was chosen for the study due to the fact that drip irrigation is adopted by the farmers to cultivate both perennial and orchard crops such as coconut, arecanut, banana, grapes, etc. According to the report of Department of Agriculture, Government of Tamil Nadu, A sample size of 400 (approximately 10 percent) of the population was taken into consideration for this study.

\section{Framework of Analysis}

The primary data collected were analyzed by using the following statistical tools.Apart from the descriptive statistical analysis, the tools like Chi-Square Test, Five Point Scaling, Average Score Analysis, Regression Analysis ANOVA,

Paired

$\mathrm{t}$-Test, and Crosstab Analysis have been used in the present study.

\section{Limitations of the Study}

The necessary precautions were taken by the researcher to prevent shortcomings. In spite of that, the present study is subject to the following limitations. First, the results of the present study may not be applicable to other blocks having different socio - economic conditions.

\section{Review of Literature}

Gupta A.J, et al., (2010), in their study, capsicum var. Nishat-1 was grown under drip irrigation with fertigation. Surface irrigation and manual fertilizer application were treated as control. The results revealed that there was significant improvement in yield, quality, water and fertilizer use efficiencies of capsicum under drip irrigation and fertigation. However, the combined effect of drip irrigation and fertigation was found superior than their individual effects. The treatment combination of 80 per cent ET through drip and 80 per cent recommended NPK through fertigation registered maximum fruit yield $(366.48 \mathrm{q} / \mathrm{ha})$. The highest water use efficiency $(29.40 \mathrm{q} / \mathrm{ha}-\mathrm{cm})$ was observed with the treatment combination of 60 per cent ET through drip +80 per cent recommended NPK through fertigation.

Suresh Kumara,P. D. and Palanisami, K. (2010)have revealed that adoption of drip irrigation technology has increased the net sown area, net irrigated area and thereby has helped in achieving higher cropping intensity and irrigation intensity. It has been found that there is a significant shift towards crops such as coconut, grapes and banana from annual crops like vegetables, sugarcane and the like. The main reasons have been found as scarcity of human labour and water. As the cropping pattern decides the adoption and suitability of drip irrigation, widespread adoption of micro - irrigation could be promoted in the regions where shift towards crops like coconut, banana and grapes are common. The analysis of economics of crop cultivation under drip and control has revealed that the drip method of irrigation has a significant impact on resources saving, cost of cultivation, yield of crops and farm profitability. The physical water and energy productivity is significantly high in drip over the flood method of irrigation.
One could conclude that the drip has a significant bearing on the private costs and benefits and hence on profit of farmers. Thus, our policy focus may be tilted towards the promotion of drip irrigation in those regions where scarcity of water and labour is alarming and where shift towards wider spaced crops is taking place.

\section{Analysis and Findings}

Table 1: Drip Irrigation Increases Yield

\begin{tabular}{|c|c|c|c|c|c|}
\hline \multicolumn{6}{|l|}{ No. of Family Members } \\
\hline None & $\begin{array}{c}10 \\
-66.7\end{array}$ & $\begin{array}{c}5 \\
-33.3\end{array}$ & $\begin{array}{l}0 \\
0\end{array}$ & $\begin{array}{l}0 \\
0\end{array}$ & $\begin{array}{c}15 \\
-100\end{array}$ \\
\hline One & $\begin{array}{c}260 \\
-75.4 \\
\end{array}$ & $\begin{array}{c}73 \\
-21.2 \\
\end{array}$ & \begin{tabular}{|c|}
10 \\
-2.9 \\
\end{tabular} & $\begin{array}{c}2 \\
-0.6 \\
\end{array}$ & $\begin{array}{c}345 \\
-100 \\
\end{array}$ \\
\hline Two & $\begin{array}{c}21 \\
-72.4\end{array}$ & $\begin{array}{c}7 \\
-24.1\end{array}$ & \begin{tabular}{|l|}
0 \\
0
\end{tabular} & $\begin{array}{c}1 \\
-3.4\end{array}$ & $\begin{array}{c}29 \\
-100\end{array}$ \\
\hline More than two & $\begin{array}{c}5 \\
-83.3 \\
\end{array}$ & $\begin{array}{c}1 \\
-16.7 \\
\end{array}$ & $\begin{array}{l}0 \\
0 \\
\end{array}$ & $\begin{array}{l}0 \\
0 \\
\end{array}$ & $\begin{array}{c}6 \\
-100 \\
\end{array}$ \\
\hline Total & $\begin{array}{c}296 \\
-74.9\end{array}$ & \begin{tabular}{|c|}
86 \\
-21.8 \\
\end{tabular} & \begin{tabular}{|c|}
10 \\
-2.5 \\
\end{tabular} & $\begin{array}{c}3 \\
-0.8\end{array}$ & $\begin{array}{r}395 \\
-100 \\
\end{array}$ \\
\hline \multicolumn{6}{|l|}{ No. of Electric Service } \\
\hline One & $\begin{array}{c}269 \\
-74.5\end{array}$ & $\begin{array}{c}80 \\
-22.2\end{array}$ & \begin{tabular}{|c|}
10 \\
-2.8
\end{tabular} & $\begin{array}{c}2 \\
-0.6\end{array}$ & $\begin{array}{c}361 \\
-100\end{array}$ \\
\hline Two & $\begin{array}{c}23 \\
-85.2 \\
\end{array}$ & $\begin{array}{c}4 \\
-14.8 \\
\end{array}$ & \begin{tabular}{|l|}
0 \\
0 \\
\end{tabular} & $\begin{array}{l}0 \\
0 \\
\end{array}$ & $\begin{array}{c}27 \\
-100 \\
\end{array}$ \\
\hline More than two & $\begin{array}{c}4 \\
-57.1\end{array}$ & $\begin{array}{c}2 \\
-28.6\end{array}$ & $\begin{array}{l}0 \\
0\end{array}$ & $\begin{array}{c}1 \\
-14.3\end{array}$ & $\begin{array}{c}7 \\
-100\end{array}$ \\
\hline Total & $\begin{array}{c}296 \\
-74.9 \\
\end{array}$ & \begin{tabular}{|c|}
86 \\
-21.8 \\
\end{tabular} & $\begin{array}{c}10 \\
-2.5 \\
\end{array}$ & $\begin{array}{c}3 \\
-0.8 \\
\end{array}$ & $\begin{array}{r}395 \\
-100 \\
\end{array}$ \\
\hline \multicolumn{6}{|l|}{ Type of Crop Cultivated } \\
\hline Standard Crops & $\begin{array}{c}32 \\
-71.1\end{array}$ & $\begin{array}{c}13 \\
-28.9\end{array}$ & $\begin{array}{l}0 \\
0\end{array}$ & $\begin{array}{l}0 \\
0\end{array}$ & \begin{tabular}{|c|}
45 \\
-100 \\
\end{tabular} \\
\hline Seasonal Crops & $\begin{array}{c}87 \\
-73.7\end{array}$ & $\begin{array}{c}25 \\
-21.2\end{array}$ & \begin{tabular}{|c|}
5 \\
-4.2 \\
\end{tabular} & $\begin{array}{c}1 \\
-0.8\end{array}$ & $\begin{array}{c}118 \\
-100 \\
\end{array}$ \\
\hline Regular Crops & $\begin{array}{c}44 \\
-86.3 \\
\end{array}$ & $\begin{array}{c}6 \\
-11.8 \\
\end{array}$ & \begin{tabular}{|l|}
0 \\
0 \\
\end{tabular} & $\begin{array}{c}1 \\
-2 \\
\end{array}$ & $\begin{array}{c}51 \\
-100 \\
\end{array}$ \\
\hline Standard \& Regular Crops & $\begin{array}{c}75 \\
-80.6 \\
\end{array}$ & $\begin{array}{c}17 \\
-18.3\end{array}$ & $\begin{array}{l}0 \\
0\end{array}$ & $\begin{array}{c}1 \\
-1.1\end{array}$ & $\begin{array}{c}93 \\
-100 \\
\end{array}$ \\
\hline Standard \& Seasonal Crops & $\begin{array}{c}58 \\
-65.9\end{array}$ & $\begin{array}{c}25 \\
-28.4\end{array}$ & \begin{tabular}{|c|}
5 \\
-5.7 \\
\end{tabular} & $\begin{array}{l}0 \\
0\end{array}$ & $\begin{array}{c}88 \\
-100 \\
\end{array}$ \\
\hline Total & $\begin{array}{c}296 \\
-74.9\end{array}$ & $\begin{array}{c}86 \\
-21.8\end{array}$ & \begin{tabular}{|c|}
10 \\
-2.5
\end{tabular} & $\begin{array}{c}3 \\
-0.8\end{array}$ & $\begin{array}{c}395 \\
-100\end{array}$ \\
\hline
\end{tabular}

From the results, it is clear that, the farmers strongly agree on the fact that drip irrigation increases crop yield after its adoption. However, they have strongly disagreed on the fact that drip irrigation controls pests and diseases.

Table 2: Chi - Square Analysis: Agricultural Profile and Opinion that Drip Irrigation Increases Yield

\begin{tabular}{|c|c|c|c|}
\hline Factors & $\begin{array}{c}\text { Chi-square } \\
\text { Value }\end{array}$ & df & $\begin{array}{c}\mathrm{p}- \\
\text { value }\end{array}$ \\
\hline Farmers Involvement in Agriculture & $10.790^{\mathrm{a}}$ & 9 & .290 \\
\hline Type of Farmers & $6.783^{\mathrm{a}}$ & 6 & .341 \\
\hline Experience in Agriculture & $12.640^{\mathrm{a}}$ & 6 & .049 \\
\hline $\begin{array}{c}\text { Number of Family Members Involved } \\
\text { in Agriculture }\end{array}$ & $5.869^{\mathrm{a}}$ & 9 & .753 \\
\hline Number of Electric Service & $19.667^{\mathrm{a}}$ & 6 & $.003^{*}$ \\
\hline Type of Crop Cultivated & $19.763^{\mathrm{a}}$ & 12 & .072 \\
\hline
\end{tabular}

Note1: a. 10 cells (50.0)) have expected count less than 5.

Note2: * indicates significant at five per cent level.

Note3: The minimum expected count is .34 . 


\section{International Journal of Science and Research (IJSR) \\ ISSN (Online): 2319-7064 \\ Index Copernicus Value (2013): 6.14 | Impact Factor (2015): 6.391}

However, the chi - square results reveal that, except the number of electric service used, all other agricultural variables have significant influence the on opinion that drip irrigation increases crop yield.

Table 3: Drip Irrigation Controls Pests and Diseases

\begin{tabular}{|c|c|c|c|c|c|c|c|c|c|c|c|c|c|}
\hline \multirow{3}{*}{$\begin{array}{c}\text { Involvement in } \\
\text { Agriculture }\end{array}$} & \multirow{2}{*}{\multicolumn{5}{|c|}{ Drip Controls Pests and Diseases }} & \multirow{3}{*}{ Total } & \multirow[b]{2}{*}{ Total } & \multirow{3}{*}{\begin{tabular}{|c|}
38 \\
-9.6 \\
\end{tabular}} & \multirow[b]{2}{*}{$\begin{array}{c}103 \\
-26.1 \\
\end{array}$} & \multirow{3}{*}{$\begin{array}{c}36 \\
-9.1 \\
\end{array}$} & \multirow{2}{*}{\begin{tabular}{|c|}
201 \\
-50.9 \\
\end{tabular}} & \multirow{2}{*}{$\begin{array}{c}17 \\
-4.3 \\
\end{array}$} & \multirow{2}{*}{$\begin{array}{r}395 \\
-100 \\
\end{array}$} \\
\hline & & & & & & & & & & & & & \\
\hline & SA & A & $\frac{N}{25}$ & DA & $\frac{\text { SDA }}{9}$ & & & & & & & & \\
\hline
\end{tabular}

\begin{tabular}{|c|c|c|c|c|c|c|c|} 
By tradition & -9.5 & -17.5 & -9.9 & -59.5 & -3.6 & -100 & \multirow{2}{*}{$\begin{array}{l}\text { From the results, it is clear that, the farmers strongly agree } \\
\text { on the fact that drip irrigation controls pests and diseases }\end{array}$} \\
\cline { 1 - 6 } Nature of job & 1 & 17 & 0 & 0 & 0 & 18 & \\
& -5.6 & -94.4 & 0 & 0 & 0 & -100 & after its adoption.
\end{tabular}

\begin{tabular}{c|c|c|c|c|c|c|} 
Self Interest & 4 & 17 & 3 & 25 & 3 & 52 \\
& -7.7 & -32.7 & -5.8 & -48.1 & -5.8 & -100 \\
\hline \multirow{2}{*}{ Income potential } & 9 & 25 & 8 & 26 & 5 & 73
\end{tabular}

\begin{tabular}{|c|cccc|c|c|c|} 
Income potential & -12.3 & -34.2 & -11 & -35.6 & -6.8 & -100 \\
\hline \multirow{2}{*}{ Total } & $\mathbf{3 8}$ & $\mathbf{1 0 3}$ & $\mathbf{3 6}$ & $\mathbf{2 0 1}$ & $\mathbf{1 7}$ & $\mathbf{3 9 5}$ \\
& $\mathbf{- 9 . 6}$ & $\mathbf{- 2 6 . 1}$ & $\mathbf{- 9 . 1}$ & $\mathbf{- 5 0 . 9}$ & $\mathbf{- 4 . 3}$ & $\mathbf{- 1 0 0}$ \\
\hline Type of & \multicolumn{2}{|c|}{ Drip Controls Pests \& Diseases } & \multirow{2}{*}{ Total } \\
\cline { 2 - 6 } Farmers & $\mathbf{S A}$ & $\mathbf{A}$ & $\mathbf{N}$ & $\mathbf{D A}$ & $\mathbf{S D A}$ & \\
\hline \multirow{2}{*}{ Marginal farmers } & 17 & 31 & 8 & 40 & 2 & 98 \\
& -17.3 & -31.6 & -8.2 & -40.8 & -2 & -100 \\
\hline \multirow{2}{*}{ Small farmers } & 10 & 31 & 17 & 108 & 7 & 173 \\
\hline
\end{tabular}

\begin{tabular}{|c|c|c|c|c|c|c|}
\hline \multirow{2}{*}{ Small farmers } & 10 & 31 & 17 & 108 & 7 & 173 \\
& -5.8 & -17.9 & -9.8 & -62.4 & -4 & -100 \\
\hline \multirow{2}{*}{ Big farmers } & 11 & 41 & 11 & 53 & 8 & 124 \\
& -8.9 & -33.1 & -8.9 & -42.7 & -6.5 & -100 \\
\hline
\end{tabular}

\begin{tabular}{|c|c|c|c|c|c|c|}
\multirow{2}{*}{ Big farmers } & 11 & 41 & 11 & 53 & 8 & 124 \\
& -8.9 & -33.1 & -8.9 & -42.7 & -6.5 & -100 \\
\hline \multirow{2}{*}{ Total } & $\mathbf{3 8}$ & $\mathbf{1 0 3}$ & $\mathbf{3 6}$ & $\mathbf{2 0 1}$ & $\mathbf{1 7}$ & $\mathbf{3 9 5}$ \\
& $\mathbf{- 9 . 6}$ & $\mathbf{- 2 6 . 1}$ & $\mathbf{- 9 . 1}$ & $\mathbf{- 5 0 . 9}$ & $\mathbf{- 4 . 3}$ & $\mathbf{- 1 0 0}$ \\
\hline Experience in & \multicolumn{2}{|c|}{ Drip Controls Pests\& Diseases } & \multirow{2}{*}{ Total } \\
\cline { 2 - 6 } Agriculture & $\mathbf{S A}$ & $\mathbf{A}$ & $\mathbf{N}$ & $\mathbf{D A}$ & $\mathbf{S D A}$ & \\
\hline \multirow{2}{*}{ Less than 5 yrs. } & 2 & 2 & 0 & 3 & 1 & 8 \\
& -25 & -25 & 0 & -37.5 & -12.5 & -100 \\
\hline \multirow{2}{*}{$5-10$ yrs. } & 12 & 38 & 11 & 128 & 7 & 196 \\
& -6.1 & -19.4 & -5.6 & -65.3 & -3.6 & -100 \\
\hline \multirow{2}{*}{ More than 10 yrs. } & 24 & 63 & 25 & 70 & 9 & 191 \\
& -12.6 & -33 & -13.1 & -36.6 & -4.7 & -100 \\
\hline \multirow{2}{*}{ Total } & $\mathbf{3 8}$ & $\mathbf{1 0 3}$ & $\mathbf{3 6}$ & $\mathbf{2 0 1}$ & $\mathbf{1 7}$ & $\mathbf{3 9 5}$ \\
\hline
\end{tabular}

\begin{tabular}{|c|c|c|c|c|c|c|}
\hline & & & & & & \\
\hline Total & $\begin{array}{c}38 \\
-9.6\end{array}$ & $\begin{array}{c}103 \\
-26.1\end{array}$ & $\begin{array}{c}36 \\
-9.1\end{array}$ & $\begin{array}{c}201 \\
-50.9 \\
\end{array}$ & $\begin{array}{c}17 \\
-4.3\end{array}$ & \multirow{3}{*}{$\begin{array}{c}395 \\
-100 \\
\text { Total }\end{array}$} \\
\hline \multirow{2}{*}{$\begin{array}{c}\text { No. of family } \\
\text { members }\end{array}$} & \multicolumn{5}{|c|}{ Drip Increases Yield } & \\
\hline & SA & $\mathbf{A}$ & $\mathbf{N}$ & DA & SDA & \\
\hline \multirow{2}{*}{ None } & 1 & 13 & 1 & 0 & 0 & 15 \\
\hline & -6.7 & -86.7 & -6.7 & 0 & 0 & -100 \\
\hline \multirow{2}{*}{ One } & 30 & 78 & 31 & 191 & 15 & 345 \\
\hline & -8.7 & -22.6 & -9 & -55.4 & -4.3 & -100 \\
\hline \multirow{2}{*}{ Two } & 4 & 9 & 4 & 10 & 2 & 29 \\
\hline & -13.8 & -31 & -13.8 & -34.5 & -6.9 & -100 \\
\hline \multirow{2}{*}{ More than two } & 3 & 3 & 0 & 0 & 0 & 6 \\
\hline & -50 & -50 & 0 & 0 & 0 & -100 \\
\hline \multirow{2}{*}{ Total } & 38 & 103 & 36 & 201 & 17 & 395 \\
\hline & -9.6 & -26.1 & -9.1 & -50.9 & -4.3 & -100 \\
\hline \multirow{2}{*}{$\begin{array}{c}\text { Number of } \\
\text { Electric Service }\end{array}$} & \multicolumn{5}{|c|}{ Drip Controls Pests and Diseases } & \multirow[t]{2}{*}{ Total } \\
\hline & $\mathbf{S A}$ & $\mathbf{A}$ & $\mathbf{N}$ & DA & SDA & \\
\hline \multirow{2}{*}{ One } & 26 & 91 & 35 & 192 & 17 & 361 \\
\hline & -7.2 & -25.2 & -9.7 & -53.2 & -4.7 & -100 \\
\hline \multirow{2}{*}{ Two } & 9 & 9 & 1 & 8 & 0 & 27 \\
\hline & -33.3 & -33.3 & -3.7 & -29.6 & 0 & -100 \\
\hline \multirow{2}{*}{ More than Two } & 3 & 3 & 0 & 1 & 0 & 7 \\
\hline & -42.9 & -42.9 & 0 & -14.3 & 0 & -100 \\
\hline \multirow{2}{*}{ Total } & 38 & 103 & 36 & 201 & 17 & 395 \\
\hline & -42.9 & -26.1 & -9.1 & -50.9 & -4.3 & -100 \\
\hline \multirow{2}{*}{$\begin{array}{l}\text { Type of Crop } \\
\text { Cultivated }\end{array}$} & \multicolumn{5}{|c|}{ Drip Controls Pests and Diseases } & \multirow[t]{2}{*}{ Total } \\
\hline & SA & A & $\mathbf{N}$ & DA & SDA & \\
\hline \multirow{2}{*}{ Standard Crops } & 4 & 4 & 2 & 34 & 1 & 45 \\
\hline & -8.9 & -8.9 & -4.4 & -75.6 & -2.2 & -100 \\
\hline \multirow{2}{*}{ Seasonal Crops } & 7 & 27 & 13 & 66 & 5 & 118 \\
\hline & -5.9 & -22.9 & -11 & -55.9 & -4.2 & -100 \\
\hline
\end{tabular}


Table 5: Paired t-test - Crop Yield between Surface and

Drip Irrigation

\begin{tabular}{|c|c|c|c|c|c|}
\hline Crop & Irrigation & Mean & $\mathrm{t}$ value & $\mathrm{df}$ & p-value \\
\hline \multirow{2}{*}{ Coconut } & Surface Irrigation & 97.30 & \multirow{2}{*}{17.17} & \multirow{2}{*}{154} & \multirow{2}{*}{$0.000^{*}$} \\
\hline & Drip Irrigation & 109.35 & & & \\
\hline \multirow{2}{*}{ Arecanut } & Surface Irrigation & 6.24 & \multirow{2}{*}{22.213} & \multirow{2}{*}{65} & \multirow{2}{*}{$0.000^{*}$} \\
\hline & Drip Irrigation & 8.52 & & & \\
\hline \multirow{2}{*}{ Grapes } & Surface Irrigation & 5.85 & \multirow{2}{*}{16.845} & \multirow{2}{*}{44} & \multirow{2}{*}{$0.000^{\circ}$} \\
\hline & Drip Irrigation & 7.73 & & & \\
\hline \multirow{2}{*}{ Banana } & Surface Irrigation & 851.61 & \multirow{2}{*}{29.676} & \multirow{2}{*}{117} & \multirow{2}{*}{$0.000^{\circ}$} \\
\hline & Drip Irrigation & 964.25 & & & \\
\hline \multirow{2}{*}{ Turmeric } & Surface Irrigation & 8.06 & \multirow{2}{*}{15.133} & \multirow{2}{*}{114} & \multirow{2}{*}{$0.000^{*}$} \\
\hline & Drip Irrigation & 10.59 & & & \\
\hline \multirow{2}{*}{ Sugarcane } & Surface Irrigation & 6.75 & \multirow{2}{*}{4.013} & \multirow{2}{*}{3} & \multirow{2}{*}{$0.028^{\prime}$} \\
\hline & Drip Irrigation & 9.08 & & & \\
\hline \multirow{2}{*}{ Onion } & Surface Irrigation & 5.65 & \multirow{2}{*}{42.293} & \multirow{2}{*}{110} & \multirow{2}{*}{$0.000^{*}$} \\
\hline & Drip Irrigation & 7.48 & & & \\
\hline \multirow{2}{*}{ Other Crops } & Surface Irrigation & 7.55 & \multirow{2}{*}{20.163} & \multirow{2}{*}{83} & \multirow{2}{*}{$0.000^{\circ}$} \\
\hline & Drip Irrigation & 9.0964 & & & \\
\hline
\end{tabular}

Source: computed from data

The above table shows the actual yield obtained in one acre of land. Coconut crop obtains mean yield of 97.3 coconuts through surface irrigation and obtains 109.35 coconuts through drip irrigation. Similarly, while noticing for all other crops drip irrigation yield is better than surface irrigation. Further, this objective tries to measure the significant impact on crop. The table shows the paired mean difference and its corresponding mean value and $p$-value. The statistical significance is tested at 5 per cent level and it is also observed that all p-values are significant at 5 per cent level.

Hence, it is justified that significant difference is found between surface irrigation and drip irrigation. It is also found that the drip irrigation system is more effective than surface irrigation in all eight crops considered for this study. Thus this analysis confirms that the drip irrigation system may be considered for better yield.

\section{Conclusion}

The results of the study reveal that the drip irrigation system, besides having water saving potential, have other advantages also like increase in crop yield, controls pests and dieses requiring less labour, requiring no personal attention, facility in application of fertilizers, etc. Thus, the drip system is very much agreeable to the farmers in the study area and hence it may be recommended to the farmers in other areas too.

\section{References}

[1] Gupta A.J, Ahmad M. Feza, Bhat F.N, (2010) ${ }^{7}$, Studies on yield, Quality, Water and Fertilizer use Efficiency of Capsicum under Drip Irrigation and Fertigation", Indian Journal of Horticulture, Vol. 67, Issue: 2, pp. $213-218$.

[2] Suresh Kumara D. and PalanisamiK. (2010) ${ }^{8}$ Impact of Drip Irrigation on Farming System: Evidence from Southern India", Agricultural Economics Research ReviewVol. 23, July-December, pp 265-272. 